\title{
Extra abdominal desmoid tumor (fibromatosis) of the breast
}

\section{loannidis C}

IASO Hospital Group, Athens, Greece

\section{Correspondence}

IASO General Hospital, 264 Mesogeion Avenue, GR-15562, Athens, Greece, E-mail: ioannidc@otenet.gr

\section{Abstract}

Desmoid tumors (or fibromatosis) of the breast are very rare findings. Their clinical and imaging features can easily mislead diagnosis, the lesions being diagnosed as breast cancer. The case of a 46-year old female patient, who presented a desmoid tumor in the area of a previously excised accessory breast, is reported. A review of the international literature revealed no other similar case. The differential diagnosis and current opinion on treatment of these tumors is discussed.

Key words: Desmoid tumor, fibromatosis, breast, surgery, breast cancer

\section{Introduction}

Extra abdominal desmoid tumors are rare soft tissue neoplasms occurring mainly in the extremities (32\%) ${ }^{1}$. Desmoid tumor (or fibromatosis) of the breast is a very rare, benign fibroblastic tumor with a tendency to recur. Its etiology remains unclear. Trauma, previous breast surgery and augmentation mammoplasty (with saline or silicone implants) have been associated with the appearance of a desmoid in the breast ${ }^{2,3}$. We report the case of a 46year old female patient who presented with a desmoid tumor of the right breast 15 months after surgical excision of an accessory breast.

\section{Case Report}

A 46-year old female patient presented with a right accessory breast requesting its excision as well as a bilateral breast reduction because of megalomastia and asymmetry (right breast larger than left). Routine mammographic and ultrasound imaging showed no abnormalities. An inferior pedicle reduction mammoplasty, a correction of the asymmetry and surgical excision of the right accessory breast was performed under general anesthesia. The postoperative course was uneventful and the histopathological examination of the resected breast tissue specimens revealed no abnormalities. Fourteen months later the patient presented with a painful, hard mass in the distal end of the upper, lateral quadrant of the right breast (toward the axilla). The mass had been growing rapidly and was causing her considerable discomfort. On clinical examination a ca $2 \mathrm{~cm}$ large, tender, firm to hard, ill-defined, mobile mass was noted ca $2 \mathrm{~cm}$ caudal to the acces- 

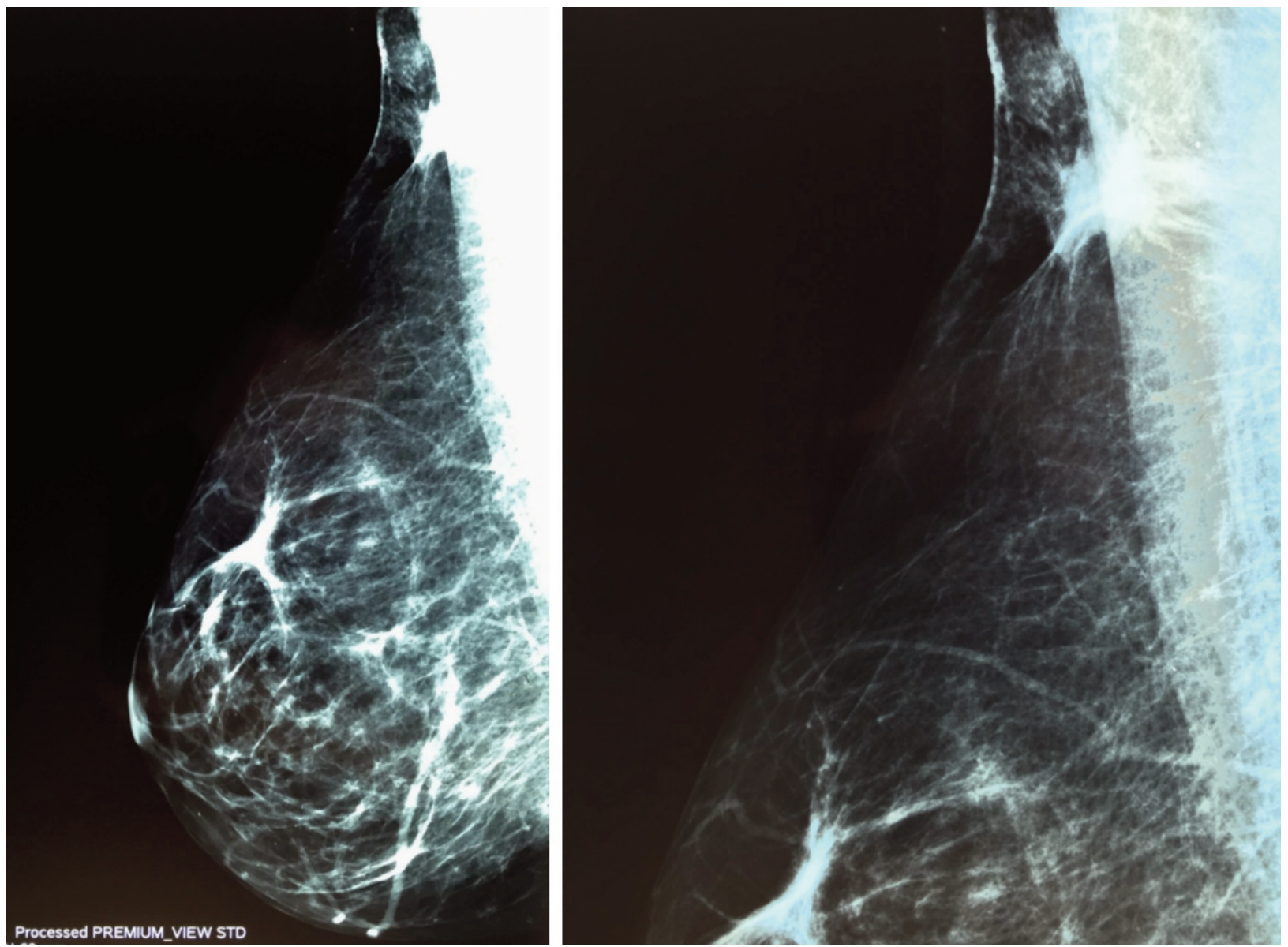

Figure 1. A. An irregular, spiculated mass is visible at the right upper end of the mammogram of the right breast. B. Close-up showing the mass more clearly.

sory breast excision scar. Mammographic imaging showed an irregular, spiculated mass in the upper, lateral quadrant of the right breast (BI RADS 4b) (Figure 1). Ultrasound imaging showed a $1.9 \mathrm{~cm}$ hypoechoic mass with irregular margins and peripheral vascularization (Figure 2). On elastography the mass proved hard $(E=4)$. The strong suspicion of malignancy dictated a wide surgical excision of the mass and surrounding tissue, which was carried out under general anesthesia. Macroscopic histopathological evaluation showed a whitish, stellate lesion with a maximum diameter of $1.2 \mathrm{~cm}$. The distance to the chest wall was $0.35 \mathrm{~cm}$ and to the overlying skin $0.15 \mathrm{~cm}$. Microscopically, the lesion was composed of intersecting bundles of bland-appearing fibroblasts with moderate cellularity, no cellular atypia, no mitotic activity or necrosis (Figure 3). The cellular proliferation index Ki67 ranged from 1 to 2\% (Figure 4). Immunohistochemically there was negativity for CD 34, S 100, Leu 7, Bcl 2, AE1/AE3 and a positive expression for SMA, actin and desmin (Figure 5). The histologic features were typical of an extra abdominal desmoid tumor. The patient has been followed for three years without any sign of a recurrence. 


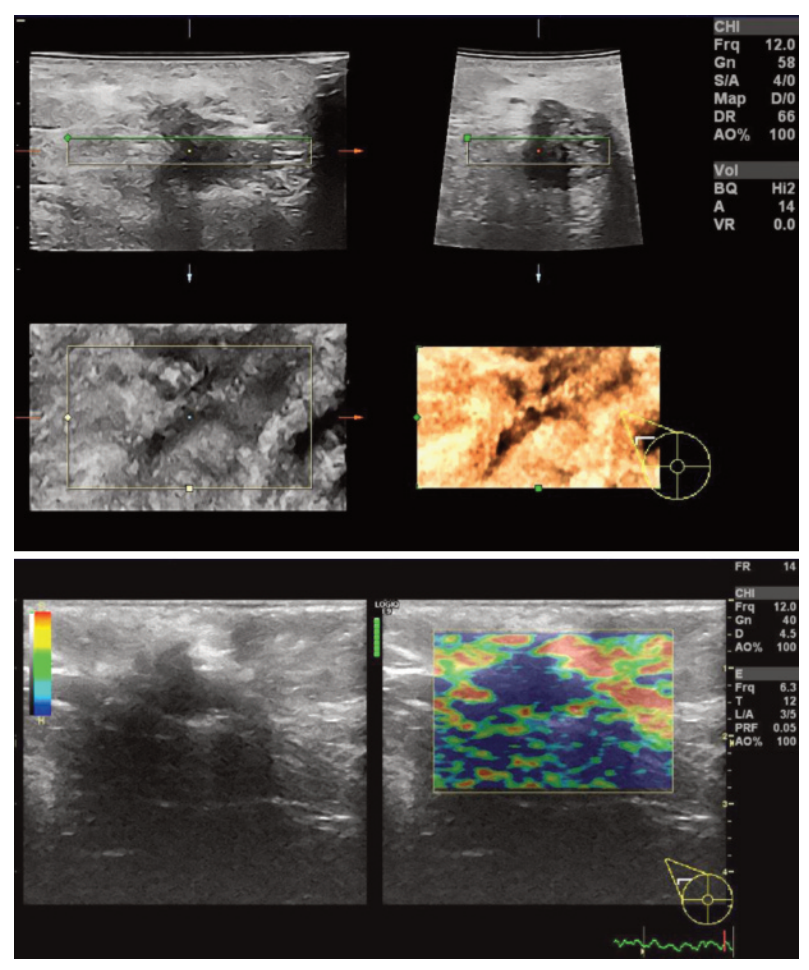

Figure 2. Ultra-sonogram showing a hypoechoic mass with A. irregular borders and B. peripheral vascularization.

\section{Discussion}

Desmoid tumors (or deep fibromatosis) are rare tumors accounting for only $0.03 \%$ of all neoplasms. They originate in musculoaponeurotic tissues and they mainly occur during the 4th and 5th decades of life (median 36 years in a large series of 211 patients $^{4}$ ). They are divided into intra-abdominal, abdominal and extra-abdominal desmoids. They all share the same morphology but differ in their presentation according to the site of development ${ }^{2}$. Intraabdominal and abdominal desmoids can develop throughout the abdomen or the abdominal wall in $12 \%$ to $38 \%$ of patients with familial adenomatous polyposis (FAP) ${ }^{3}$ Extra abdominal desmoids most frequently occur in the extremities ${ }^{4}$.

Desmoids of the breast constitute less than $0.2 \%$ of all breast tumors ${ }^{5}$. They usually involve the underlying fibroaponeurotic fascia; a desmoid confined to

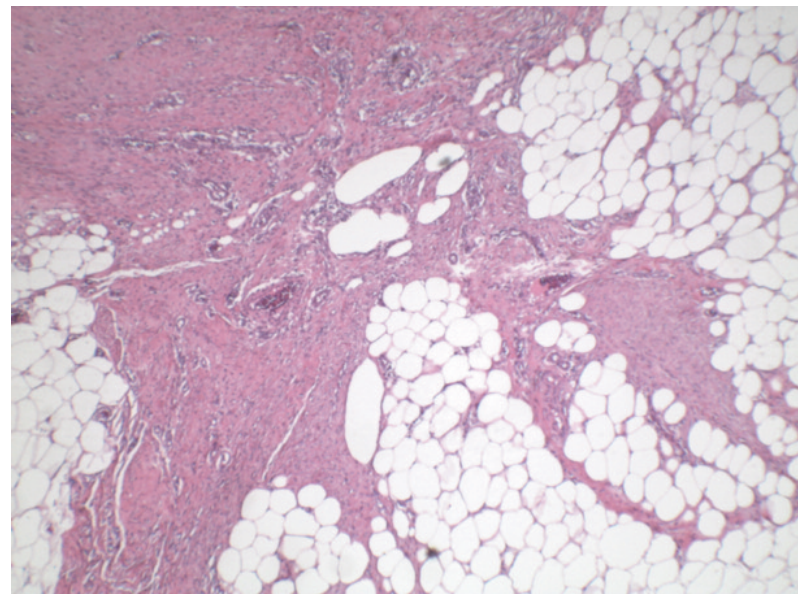

Figure 3. Micrograph showing intersecting bundles of blandappearing fibroblasts with moderate cellularity. Neither atypia nor mitotic activity or necrosis, are seen (HxE X 40).

the breast is a rare lesion ${ }^{6}$. Most desmoids of the breast present as firm to hard, usually discrete masses, however, a few are "ill defined" or "poorly circumscribed" ${ }^{\prime \prime}$, as was the case presented here. Skin dimpling over the lesion or tethering of the tumor to the skin may be present ${ }^{5}$.

The cause of breast desmoid tumors is not well understood. They have been associated with previous surgery (saline and silicone breast implants intact or ruptured, fibro adenoma excision, partial or total mastectomy for cancer) $)^{5,7,8,9}$, trauma ${ }^{5}$, oral contraceptives ${ }^{5}$ and chemotherapy ${ }^{5}$. The present case is, to the best of our knowledge, the first reported to have occurred after surgical excision of an accessory breast and chemotherapy for gynecological cancer.

Most cases are reported in women, though there have been a few cases encountered in male patients ${ }^{7}$. Unlike abdominal desmoid tumors, mammary fibromatosis does not appear to be associated with pregnancy. Median age of patients with breast desmoids at presentation was 45 years (range, 2276 years) in one of the few published series of 32 patients ${ }^{10}$. Tumors presenting after previous sur- 


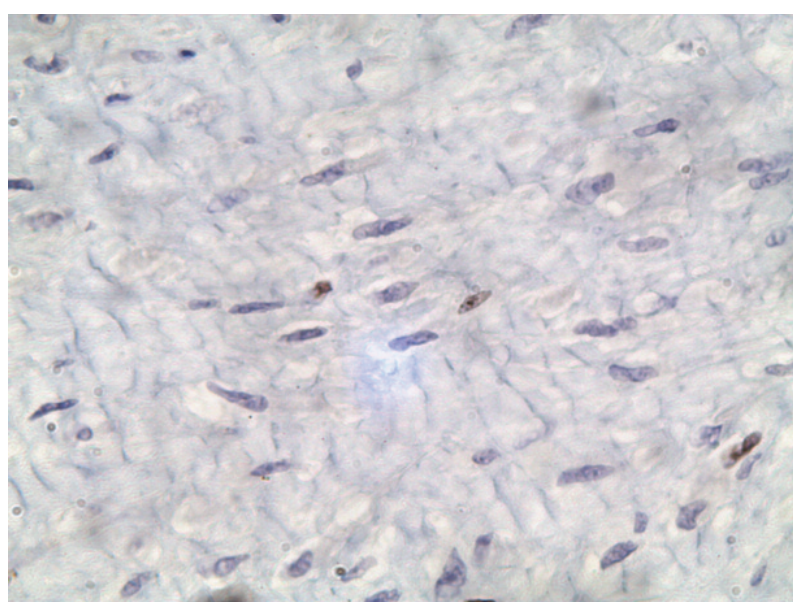

Figure 4. Nuclear positivity against Ki67 was noticed in 1-2\% of the fibroblasts (x 400).

gery were diagnosed at a median of 24 months postoperatively ${ }^{10}$. The patient described here first noticed her lesion, and immediately seeked medical consultation, 14 months after excision of the accessory breast.

Mammographically, desmoids are often irregularly shaped, uncalcified, high density masses with spiculated margins that mimic breast cancer (B1-RADS category 5$)^{6,7,11}$. However, mammography visualized the mass in only $38 \%$ of cases in the series reported by Neuman et $\mathrm{al}^{10}$. Sonographically, they appear as solid, spiculated or microlobulated, hypoechoic masses with a thick echogenic rim and posterior attenuation $^{6}$. MRI is the best imaging technique for evaluating tumor extent, in particular chest wall involvement ${ }^{7}$. The masses are typically irregular and isotense to muscle on T1-weighted images. On T2weighted images they present variable high signal intensity ${ }^{7}$.

Several authors propose that all patients should undergo a planned biopsy (by radiologically guided core needle biopsy ${ }^{12}$ ). Others, however, support the notion that a biopsy may not be needed when the lesion is small $(<2 \mathrm{~cm})$ or when it is a superficial mass that can be completely removed with negative mar-

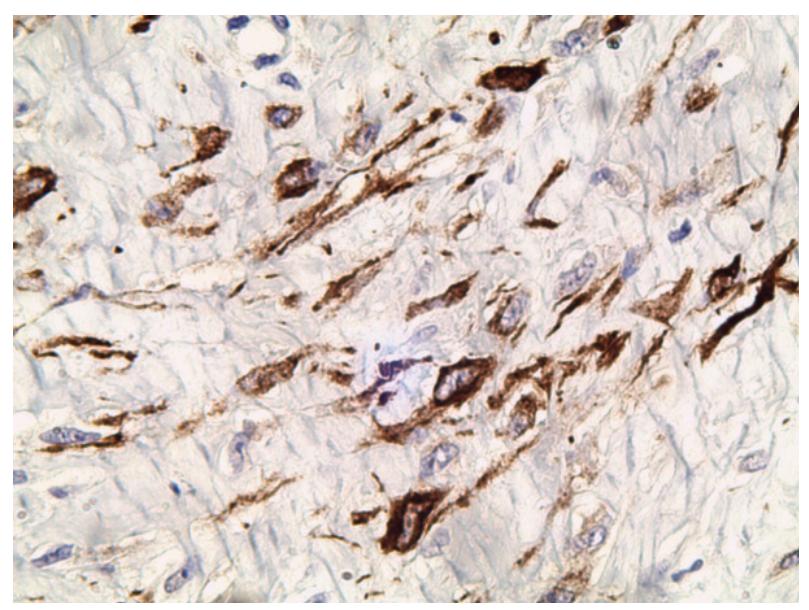

Figure 5. Immunohistochemically, there was a positive expression of desmin (x 400).

gins by excisional biopsy ${ }^{13}$. We agree with the latter opinion and have therefore performed an excisional biopsy, which sets the final diagnosis and at the same time offers definitive treatment.

Complete excision with clear margins is the currently accepted mode of treatment. When the chest wall is infiltrated, a resection of chest wall with rib cage may be necessary to complete excision ${ }^{7}$ resulting, however, in undesired disfigurement. Recurrence rates are high and have been reported between $25 \%{ }^{14}$ and $29 \%{ }^{10}$. A trend toward more frequent recurrences was observed in patients with positive versus negative margins and those with larger tumors ${ }^{10}$. The infiltrative nature of desmoids necessitates documentation of tissue margins in order to reduce the possibility of recurrences ${ }^{14}$. Younger age is another predisposing factor for recurrence ${ }^{10}$. The latter usually occurs within 4 to 15 months of excision ${ }^{10,14}$. Breast reconstruction, whenever indicated, should be best delayed until after this period.

Adjuvant anti hormonal therapy and chemotherapy has both been administered with variable success. Postoperative radiotherapy results in significant reduction of the local recurrence rate but only in the case of involved surgical margins. Radiotherapy is not 
indicated for the treatment of gross disease; it is associated with a high complication rate, which is dose dependent ${ }^{6,15}$. Recently, Roussin et al ${ }^{16}$ proposed observation as a possible mode of treatment. These authors based their recommendation on the results of a study, which showed a reduction of the median tumor size $(-4 \mathrm{~mm}$, range -13 to $+20 \mathrm{~mm})$ after frontline observation. The encouraging results could enable the identification of patients who require loco-regional treatment, however, this strategy needs further evaluation ${ }^{16}$.

Differential diagnosis of desmoid tumors showing prominent myxoid changes should include nodular fasciitis (or any reactive myofibroblastic proliferation), scar tissue, desmoplastic fibroblastoma and neurofibroma. Cellular variants may be confused with malignant lesions. However, desmoids lack the cellular atypia, numerous and atypical mitoses, and tumor necrosis typical for fibrosarcoma or monoplastic synovial sarcoma ${ }^{2}$. Immunohistochemistry is of considerable value in setting the definitive diagnosis ${ }^{2,17}$.

In conclusion, the desmoid tumors of the breast are rare, benign, locally aggressive fibroblastic lesions, which mimic breast cancer. They have the tendency to recur, but have no metastatic potential. Early recognition and wide local excision is the treatment of choice.

\section{References}

1. Privette A, Fenton SJ, Mone MC et al: Desmoid tumor: a case of mistaken identity. Breast J 2005; 11: $60-4$.

2. Deep (desmoid-type) fibromatoses. In: Bone and soft tissue pathology. Folpe AL, Inwards CY (Eds), Churchill Livingstone, 2010, p54-8

3. Ludwig KA: Tumors of the colon and rectum. In: Conn's Current Therapy. Rakel RE, Bope ET (Eds),Saunders (Elsevier), Philadelphia, 2006, p686.
4. Peng PD, Hyder O, Mavros MN et al: Management and recurrence patterns of desmoid tumors: a multi-institutional analysis of 211 patients. Ann Surg Oncol 2012; 19: 4036-42.

5. Rosen PP, Ernsberger D: Mammary fibromatosis. A benign spindle-cell tumor with significant risk for local recurrence. Cancer 1989; 63: 1363-9.

6. Erguran- Dogan B, Dempsey PJ, Ayyar G et al: Primary desmoid tumor (extraabdominal fibromatosis) of the breast. AJR 2005; 185: 488-9.

7. Glazebrook KN, Reynolds CA: Mammary fibromatosis. AJR 2009; 193: 856-60.

8. Aaron AD, O'Mara JW, Legendre KE et al: Chest wall fibromatosis associated with silicone breast implants. Surg Oncol 1996; 5: 93-9.

9. Povoski SP, Jimenez RE: Fibromatosis (desmoid tumor) of the breast mimicking a case of ipsilateral metachronous breast cancer. World J Surg Oncol 2006; 4: 57.

10. Newman HB, Brogi E, Ebrahim A et al: Desmoid tumors (fibromatoses) of the breast: a 25- year experience. Ann Surg Oncol 2008; 15: 274-80.

11. Ebrahim L, Perry J, Taylor JB: Fibromatosis of the breast: a pictorial review of the imaging and histopathology findings. Clin Radiol 2014; 69: 1077-83.

12. Eastley N., Aujla R, Silk R et al: Extra-abdominal desmoid fibromatosis- a sarcoma unit review of practice, long term recurrence rates and survival. Eur J Surg Oncol 2014; 40: 1125-30.

13. Wilder F, D' Angelo S, Crago AM: Soft tissue tumors of the trunk: management of local disease in the breast and chest and abdominal walls. J Surg Oncol 2015; 11: 546-52

14. Wargotz ES, Norris HJ, Austin RM et al: Fibromatosis of the breast: A clinical and pathological study of 28 cases. Am J Surg Pathol 1987; 11: 38-45.

15. de Bree E, Kens R, Melissas J et al: Desmoid tumors: need for an individualized approach. Expert Rev Anticancer Ther 2009; 9: 525-35. 
16. Roussin S, Mazouni C, Rimareix F et al: Toward a new strategy in desmoid of the breast? Eur J Surg Oncol 2015; 41: 571-6.

17. Chummun S, McLean NR, Abraham $S$ et al: Desmoid tumor of the breast J Plast Reconstr

Received 27-2-2018 Aesthet Surg 2010; 63: 339-45.

Revised 18-3-2018

Accepted 28-3-2018 